УДК 37.072

\title{
В.Ю. Соколов
}

\section{СЕТЕВОЕ ВЗАИМОДЕЙСТВИЕ «ШКОЛА - ВЕДУЩИЙ ИССЛЕДОВАТЕЛЬСКИЙ УНИВЕРСИТЕТ »: ИДЕНТИФИКАЦИОННЫЕ АСПЕКТЫ}

\author{
Выполнено в рамках работ по проекту «Человек в меняющемся мире. Проблемы идентичности и социальной адаптации \\ в истории и современности» (грант Правительства РФ П 220 № 14.B25.31.0009).
}

\begin{abstract}
Рассматриваются изменения в сетевом взаимодействии учреждений общего образования и Томского государственного университета в связи с переходом последнего в статус ведущего исследовательского университета. Анализируется, как стратегия трансформации университета в инновационную корпорацию, курс на создание вокруг себя адекватного научнообразовательного сообщества потребовали перенастройки системы отношений со школами региона, актуализировали необходимость определения их места в новой кооперации.

Ключевые слова: исследовательский университет; идентификация; взаимодействие; школа.
\end{abstract}

Вопросы взаимодействия школ и вузов в последние полтора десятилетия привлекли внимание как теоретиков, так и практиков отечественного образования, специалистов в области управления образованием. К активному сотрудничеству школы и вузы подтолкнуло введение компетентностной модели образования, предполагающей трансформацию традиционных представлений об учебной деятельности, переход от трансляции предметных знаний к обучению их использования на практике. В решении этой сложной задачи учреждения среднего и высшего образования начали вступать в различные виды взаимодействия, создавая в рамках профильных классов старшей школы сетевые образовательные проекты, программы, модули.

Сетевыми эти формы взаимодействия, как известно, стали называться потому, что создавались на основе взаимовыгодных «горизонтальных» связей, минуя органы управления образованием. Вузы помогали школам квалифицированными преподавателями, программами и оборудованием, школы «поставляли» вузам хорошо подготовленных абитуриентов, в первую очередь, отличавшихся высокими показателями ЕГЭ. Заметный вклад в разработку и внедрение различных сетевых форм сотрудничества внесли томские педагоги и исследователи, особенно представители ТГУ, силами которых были созданы и описаны разнообразные прецеденты подобного взаимодействия [1], изучены проблемы, обозначены перспективы сотрудничества школ и вузов в регионе [2].

Разработка данной тематики в региональном образовании получила новый импульс в связи с изменениями в развитии двух ведущих университетов Томска государственного классического и политехнического. Изменения связаны с успешным участием вузов в проекте «5 ТОР-100», предполагающем получение статуса ведущего исследовательского университета и права бороться за высокие мировые рейтинги. Новые целевые установки подтолкнули томские вузы не только к интенсификации своей деятельности, но и к разработке своего особого пути к достижению поставленной цели, осмыслению специфики своего развития, формированию «своего» места среди наиболее успешных университетов мира. В этот процесс самоидентификации ведущих университетов уже вовле- каются и будут в дальнейшем вовлекаться различные учреждения общего образования, перед которыми возникают аналогичные задачи самоопределения. В данной публикации на примере взаимодействия ТГУ и учреждений общего образования хотелось бы обратить внимание на трудности, которые актуализируются на этом пути, и возможностях их преодоления.

Переход ТГУ в статус ведущего университета, постановка принципиально новых, чрезвычайно сложных стратегических целей актуализировали задачи поиска дополнительных ресурсов развития, разработки особых механизмов повышения конкурентоспособности, формирования нового уклада жизни и деятельности университета. Решение перечисленных задач потребовало реализации целого комплекса проектов, направленных на создание особой модели развития исследовательского университета, основанной на «перестройке базовых процессов ТГУ в логике производства инноваций» [3], «выращивании» сотрудниками новых форм своей профессиональной деятельности. В образовательной сфере в качестве фундамента внутренних корпоративных стандартов университета были заявлены принципы междисциплинарности, индивидуализации, открытости и ориентация на формирование проектных, исследовательских, инновационно-предпринимательских компетенций.

Новый курс развития и потребности в дополнительных ресурсах оказали влияние на обновление руководством ТГУ стратегии взаимодействия университета со школами региона. Опираясь на положения дорожной карты реализации проекта «5 ТОР-100», руководители блока, отвечающего за учебную работу в университете, сформулировали новые запросы к школьному образованию следующим образом: «Система общего образования рассматривается университетом не только как поставщик сильных абитуриентов с высокими показателями ЕГЭ, но и как партнер в создании системы выявления талантливых детей и молодежи, их раннего привлечения в университетскую среду для формирования “своего” абитуриента, т.е. мотивированного и подготовленного к обучению по внутренним корпоративным стандартам университета» [4. С. 3].

В.В. Дёминым и Е.А. Сухановой была заявлена готовность университета самому выступить в качестве ресурса развития региональной системы общего обра- 
зования. «Сетевое взаимодействие университета с организациями общего и дополнительного образования, органами управления образованием, - заявили они, - рассматривается как составная часть третьей роли университета, а именно как механизм формирования и развития интеллектуального ресурса региона» [4. C. 4].

Сформулировав обновленные запросы к взаимодействию с системой общего образования, руководство ТГУ закономерно оказалось перед сложной проблемой поиска новых партнеров. Сложность данной проблемы, на наш взгляд, заключается в необходимости решения, по крайней мере, двух не простых задач. Во-первых, необходимо определить и привлечь к сотрудничеству достаточно широкий круг «своих» партнеров, имеющих целевые установки и ресурсы для подготовки абитуриентов, соответствующих «внутренним корпоративным стандартам университета». А во-вторых, необходимо создать устойчивую «систему» вовлечения предполагаемых абитуриентов в университетскую среду. Фактически речь идет о том, что ТГУ, чтобы стать университетом «мирового уровня», нужны не отдельные партнеры, способные и внутренне мотивированные к участию в формировании университетом «своего» абитуриента, нужен собственный корпоративный сектор в региональной системе общего образования, ориентированный на инновационное развитие.

В определенной мере эта идея просматривается в статье уже упомянутых авторов из руководства ТГУ, в которой в качестве ближайшей перспективы указывается необходимость «сформировать вокруг университета профессиональное научно-образовательное сообщество, имеющее единое представление о задачах современного образования и способах их реализации, а также желание и готовность влиять на решение актуальных задач развития системы образования в целом» [Там же. С. 5].

Таким образом, в дополнение к уже традиционной задаче набора абитуриентов-высокобалльников в ТГУ были выдвинуты новые: набор абитуриентов, соответствующих корпоративным стандартам инновационного университета, и формирование вокруг университета сообщества учреждений региональной системы общего образования, ориентированного на инновационное развитие.

Сопоставление традиционных и новых задач в развитии взаимодействия «школа - ТГУ» невольно подталкивает к мысли, что новые задачи требуют новых стратегий и новых типов взаимодействия в указанной связке образовательных учреждений. В этой мысли особенно утверждаешься при помещении перечисленных задач в контекст типологии эмпирических моделей взаимодействия «вуз - школа», разработанной в начале 2010-х гг. профессором ТГУ Г.Н. Прозументовой. В этой типологии выделены некоторые характерные особенности взаимодействия между учреждениями общего и высшего образования по целевым установкам, системности отношений, инновационному потенциалу.

В первой группе характерными для взаимодействия образовательных учреждений являются случай- ные связи. Такое взаимодействие возникает «по ситуации», основывается преимущественно на личных контактах и характеризуется прагматичной ориентацией на повышение результатов ЕГЭ, увеличение количества учащихся, поступающих в вузы.

Во второй группе преобладает стратегия продуманного «использования друг друга» для решения каждым учреждением своих задач: взаимодействие фокусируется на решении задачи успешного поступления учащихся в вузы и «набора» абитуриентов. Поэтому школы развивают отношения с престижными вузами, создают профильные классы, что обеспечивает им не только высокий процент «поступающих», но и репутацию учреждения с высоким качеством образования. Вузы получают хорошо подготовленных учащихся и репутацию образовательного учреждения, принимающего абитуриентов с высоким баллом ЕГЭ.

Для третьей группы отношений определяющим является «поглощение» вузом школы: взаимодействие определяется своеобразным «подтягиванием» задач общего образования к задаче подготовки и успешного обучения учащихся в вузе. Такая модель часто проявляется во взаимодействии вузов и старшей ступени школы или в создании отдельных, самостоятельных образовательных учреждений при вузах (лицеи, гимназии).

Четвертую модель характеризует ориентир учреждений на «совместное производство» инновационных разработок, обеспечивающих повышение качества образования, создание новых образовательных услуг и становление образования как области инновационного развития. В таком взаимодействии ставятся общие для школы и вуза задачи совершенствования образовательного процесса, проектируются, апробируются разные модели организации образования, создаются и внедряются инновационные разработки, обеспечивающие повышение качества образования.

Пятая модель нацелена на совместное формирование «образовательного сообщества» как субъекта управления образованием в региональном образовательном пространстве. Наиболее подготовленным к такой совместной работе со стороны системы общего образования являются школы-лаборатории. При взаимодействии школ-лабораторий и вузов создаются не только инновационные разработки, повышающие качество образования, но и субъекты (группы, команды), обладающие потенциалом изменения качества образования, преодоления учрежденческих границ, «эгоизма» образовательных учреждений [5. С. 186].

Данная типология моделей взаимодействия «школа - вуз» интересна тем, что ее можно рассматривать одновременно как инструмент идентификации партнеров и как средство продуманного формирования идентификационной группы. Последнее становится возможным в силу того, что в типологии не только зафиксированы разные качества взаимодействия учреждений образования, но, что особенно актуально, четко прописан вектор движения и пошаговые ориентиры для развития этого взаимодействия: от случайных, очень «корыстных», разноцелевых связей к выработке общей стратегической линии по созданию «образовательного сообщества», объединенного задачей построения инновационного сектора в регионе. 
Сложность применения данной типологии к опыту университета связана с тем, что в настоящее время в вопросах взаимодействия ТГУ с учреждениями общего образования сложились два подхода. Первый подход, обладающий повышенной актуальностью, исходит из обязательств, которые университет как участник проекта «5 ТОР-100» взял на себя по приему абитуриентов. Согласно этим обязательствам, прописанным в «дорожной карте» программы повышения конкурентноспособности, к 2020 г. планируется довести средний балл ЕГЭ поступающих в ТГУ на бюджетные места до 76 [3]. В связи с чем особый интерес для университета представляет традиционный тип партнеров - учреждения общего образования, которые достигают особых успехов в подготовке «высокобалльников». В приведенной типологии взаимодействия «школа - вуз» эти учреждения расположены в основном во второй и третьей группах. Именно в двух указанных группах, как показывает практика набора абитуриентов, по преимуществу сосредоточен основной костяк «высокобалльников» г. Томска.

Второй подход в работе ТГУ с партнерами из системы общего образования продолжает намеченную в предыдущие годы линию сотрудничества в инновационной деятельности. Она опирается на «стратегию «совместного производства» образовательных продуктов (технологий, проектов, программ)», которые могут «повлиять на качество общего образования за счет расширения школьной программы и фокусировки форм и способов образовательной деятельности на проектно-исследовательской области» [4. С. 5]. К числу школ, уже вовлеченных в реализацию данной стратегии, в университете относят, в первую очередь, школы-лаборатории ТГУ - школа «Эврика-развития», СОШ № 49 (Школа совместной деятельности), Северская гимназия. Именно на эту группу учреждений из пятой модели указывала Г.Н. Прозументова как на самых перспективных партнеров по взаимодействию с точки зрения развития инновационного потенциала образования.

«Взаимодействие в этой модели, - писала она, выражается в сильных синергетических эффектах, которые не присущи какой-либо одной из взаимодействующих сторон, а являются именно эффектами взаимодействия, порождаются ими» [5. С. 183]. По мнению Г.Н. Прозументовой, в рамках этой модели создаются три важнейших составляющих развития потенциала взаимодействия «школа - вуз»: 1) «антропологический потенциал образования, т.е. потенциал осмысленного участия и “личного присутствия” человека в организации образования», 2) «гуманитарный потенциал, т.е. потенциал влияния сообществ на организацию образования», 3) «потенциал самоорганизации и синергизма, т.е. выделение взаимодействия, совместности как предмета образования и условия изменения его качества» [Там же. С. 187]. К сожалению, взаимодействие между университетом и школами-лабораториями в последние два-три года по разным причинам заметно ослабло, потеряло былую интенсивность и систематичность. В то же время проведенная в феврале 2016 г. в Томске Международная тьюторская конференция «Многообразие и индивиду- ализация как возможность становления образовательной культуры» с активным участием школлабораторий показала высокий инновационный потенциал развития сотрудничества университета с этими учреждениями общего образования.

Не хотелось бы вводить жестких разграничений между двумя указанными подходами, но все-таки необходимо отметить, что за ними стоят ориентации на учреждения общего образования с очень разными целевыми и ценностными установками, так как система овладения инициативными компетенциями и система «натаскивания» на успешное прохождение ЕГЭ имеют существенные различия. (Характерными в этой связи представляются результаты анализа сформированности проектных, исследовательских, коммуникативных компетенций у участников знаковых для ТГУ образовательных событий (Форсайт-сессия, Молодежный форум), проведенных весной 2016 г. Из числа приглашенных - учащиеся старших классов ведущих гимназий и лицеев г. Томска, только треть показала успешные результаты.)

Однако не только отмеченная разновекторность в отношениях с учреждениями общего образования затрудняет формирование университетом собственного корпоративного сектора инновационного образования в регионе. Следует сказать и о невнятной политике (если о таковой вообще можно говорить) самих учреждений общего образования в отношениях с ТГУ в его новом статусе. Подавляющее большинство из них продолжает традиционно рассматривать университет в основном как реципиента будущих абитуриентов, очень ограниченно используя его ресурсы в обновлении содержания школьного образования, совместной разработке новых образовательных проектов и программ, повышении квалификации педагогов и управленцев в рамках требований новых ФГОС и т.д.

Создается впечатление, что сложившиеся в отношениях ТГУ и школ региона «недопонимание» и разнонаправленность интересов, препятствующие формированию осознанной и устойчивой принадлежности к региональному инновационному сообществу, в немалой степени обусловлены дефицитом системных форм взаимодействия. Подобный дефицит выражается в отсутствии организационной основы для систематического и всестороннего обсуждения ее участниками актуальных для них ценностей и целей образования, разработки и коррекции стратегии совместного развития, подготовки инновационных проектов и программ. Представляется, что одним из средств восполнения данного дефицита могло бы стать создание силами ТГУ и его партнеров регионального Института взаимодействия учреждений общего и высшего образования, основанного на сетевых принципах. В основание такого Института могут быть положены две ключевые установки.

Во-первых, центральное место в его деятельности должна занять идея создания «образовательного сообщества», в которое могут входить различные неформальные группы и официальные учреждения, выступающие с инициативами по развитию образования в регионе. Реализация данной установки позволит задать ценностные приоритеты в деятельности парт- 
неров по Институту, сосредоточив их на задачах создания инновационного сектора в региональном образовании. (Понятно, что взаимодействие ТГУ со школами в подготовке абитуриентов-высокобалльников не снимается с повестки дня, но оно начинает приобретать другие параметры. С одной стороны, в школахлабораториях уже накоплен опыт разработки программ по подготовке к ЕГЭ с использованием современных образовательных технологий. С другой стороны, формат единого государственного экзамена в настоящее время испытывает заметную трансформацию - сокращение репродуктивных и расширение творческих заданий. Вероятно, эта тенденция в дальнейшем еще более прочно свяжет успешность окончания школы с овладением инновационными компетенциями.)

Во-вторых, все партнеры по Институту, исходя из принципов сетевого сотрудничества, должны получить право участвовать во всех формах и на всех уровнях его деятельности. Это значит, что они будут иметь возможность принять участие в разработке, реализации, управлении и исследовании основных форм инновационной деятельности по развитию образования в регионе - образовательных событий, проектов, программ, стратегий. Данная возможность неограниченного участия в работе Института одновременно становится и основанием для выбора поля деятельности его «сотрудников». Схематично эту возможность можно выразить следующим образом:

Уровни и формы взаимодействия в образовательном сообществе

\begin{tabular}{|l|l|l|l|l|}
\hline \multicolumn{1}{|c|}{ Уровни } & $\begin{array}{c}\text { Разра- } \\
\text { ботка }\end{array}$ & $\begin{array}{c}\text { Реали- } \\
\text { зация }\end{array}$ & $\begin{array}{c}\text { Управле- } \\
\text { ние }\end{array}$ & $\begin{array}{c}\text { Исследо- } \\
\text { вание }\end{array}$ \\
\hline $\begin{array}{l}\text { Образовательное } \\
\text { событие }\end{array}$ & & & & \\
\hline Образовательный проект & & & & \\
\hline $\begin{array}{l}\text { Образовательная } \\
\text { программа }\end{array}$ & & & & \\
\hline $\begin{array}{l}\text { Образовательная } \\
\text { сратегия }\end{array}$ & & & & \\
\hline
\end{tabular}

В развернутом виде информация, составленная на основе карты, даст комплексное представление о деятельности участников сообщества, об их тактических установках и предоставит возможность сделать собственный выбор относительно своего места, роли и содержания деятельности в образовательном сообществе региона. Думается, что предлагаемая матрица может послужить в качестве идентификационной карты и выполнять функцию еще одного инструмента самоопределения для установления принадлежности участника образования к инновационному сообществу.

Проводя различия в уже описанных средствах идентификации, следует сказать, что если типология первичных моделей взаимодействия «школа - вуз» Г.Н. Прозументовой может рассматриваться, прежде всего, как инструмент для выбора ценностных ориентиров, то карта определения уровней и форм взаимодействия рассчитана на «перевод» ценностных ориентиров в деятельностную форму и построение собственных «каналов входа» в региональное инновационное сообщество. Создание Института взаимодействия учреждений общего и высшего образования будет особенно актуально, если принять во внимание те бурные изменения, которые сейчас происходят как в университетской среде, так и в системе общего образования. Новые повороты в реформировании обеих систем образования, несомненно, каждый раз будут заново поднимать идентификационные проблемы, требовать перенастройки отношений в региональном инновационном сообществе и разработки более эффективных форм взаимодействия их представителей.

Институт, построенный на сетевых принципах взаимодействия, привлечет учреждения общего и высшего образования, прежде всего, возможностью самого разнообразного сотрудничества с университетом. Эта возможность в особой мере будет способствовать более успешной реализации третьей роли ТГУ по развитию интеллектуального ресурса региона, в значительной мере поможет формированию «вокруг университета профессионального научно-образовательного сообщества, имеющего единое представление о задачах современного образования и способах их реализации». Взяв курс на трансформацию своей корпоративной идентичности, на превращение в активно развивающуюся инновационную корпорацию, университет может использовать накопленный опыт взаимодействия со школами для распространения своих принципов в системе общего образования, для создания в регионе единого инновационного образовательного комплекса.

\section{ЛИТЕРАТУРА}

1. Современный университет - школа: прецеденты и феномены взаимодействия / под ред. Г.Н. Прозументовой. Томск : Изд-во Том. ун-та, 2007.

2. Взаимодействие вузов и школ для становления Открытого образовательного пространства: потенциал, проблемы, задачи управления / под ред. Г.Н. Прозументовой. Томск : ТМЛ-Пресс, 2013.

3. План мероприятий по реализации программы повышения конкурентоспособности («дорожной карты») федерального государственного автономного образовательного учреждения высшего образования «Национальный исследовательский Томский государственный университет» на 2013-2020 гг. (2-й этап - 2015-2016 гг.) Томск, 2015. URL: viu.tsu.ru

4. Демин В.В., Суханова Е.А. Сетевое взаимодействие классического исследовательского университета и системы общего образования: опыт и перспективы // Информатика и образование. 2015. № 6. С. 3-6.

5. Прозументова Г.Н. Потенциал взаимодействия вузов и школ: эмпирические модели // Вестник Томского государственного университета. 2012. № 358. C. 182-187.

Статья представлена научной редакцией «История» 11 ноября 2016 г. 
THE "SCHOOL AND LEADING RESEARCH UNIVERSITY" NETWORK INTERACTION: IDENTIFICATION ASPECTS

Vestnik Tomskogo gosudarstvennogo universiteta - Tomsk State University Journal, 2017, 414, 129-133.

DOI: $10.17223 / 15617793 / 414 / 20$

Viktor Yu. Sokolov, Tomsk State University (Tomsk, Russian Federation). E-mail: sokvu@yandex.ru

Keywords: leading research university; identification; interaction; school.

The purpose of this article is to consider changes in the network interaction of institutions of general education and Tomsk State University in connection with the latter obtaining the status of a leading research university. The sources for this article were statements in the press of the leaders of the University on the issues of network interaction of the University with institutions of general education, publications of University professors on the topic of the study and participant observation of the author of this article. Regarding the latter source, the author is the head of one of the network programs (TSU - School 49, Tomsk) and a participant of the project "Interaction of TSU with the system of general education". Networking of institutions of general and higher education have been actively developed and studied in our country since the beginning of the 2000s. The active cooperation of schools and universities was prompted by the introduction of the competence model of education, the transition from the traditional translation and reproduction of subject knowledge to the teaching of the use of knowledge in practice. Mutual interest caused network cooperation: schools needed new curricula, qualified teachers, equipment, universities needed applicants that have mastered relevant competencies. Tomsk teachers and researchers made a significant contribution to the development and implementation of various network forms of cooperation, particularly active in this direction were the staff of Tomsk State University, who described the precedents, identified the problems and outlined the prospects of cooperation between schools and universities in the region. It seems that the main factor of changes in the interaction of TSU with the institutions of general education in the last three years is the transition to transforming the University into an innovative corporation. A new course, on the one hand, demanded restructuring the system of relations of the University with schools of the region, on the other hand, actualized the problem of the identity of schools in relation to the new cooperation. However, the vast majority of schools in the region continue to traditionally consider the University as a recipient of future entrants and make a very limited use of its innovative resources. In the author's opinion, an important reason for such a "neglect" of the new role of TSU is the shortage of effective forms of self-identification, in which the University and schools could present their positions, engage in dialogue to understand each other, develop joint projects and programmes. A platform for dialogue could be set up by TSU and its partner in the form of the Regional Institute of Interaction of Institutions of General and Higher Education based on network principles. The establishment of this Institute will be particularly relevant and promising, taking into account the rapid changes that are taking place within the University environment and in the general education system. These changes at every turn will re-raise identification problems.

\section{REFERENCES}

1. Prozumentova, G.N. (ed.) (2007) Sovremennyy universitet - shkola: pretsedenty i fenomeny vzaimodeystviya [Modern university - school: precedent phenomena and interactions]. Tomsk: Tomsk State University.

2. Prozumentova, G.N. (ed.) (2013) Vzaimodeystvie vuzov i shkol dlya stanovleniya Otkrytogo obrazovatel'nogo prostranstva: potentsial, problemy, zadachi upravleniya [Interaction of universities and schools for the development of open educational space: potential problems and tasks of management]. Tomsk: TML-Press.

3. Tomsk State University. (2015) Plan meropriyatiy po realizatsii programmy povysheniya konkurentosposobnosti ("dorozhnoy karty”) federal'nogo gosudarstvennogo avtonomnogo obrazovatel'nogo uchrezhdeniya vysshego obrazovaniya "Natsional'nyy issledovatel'skiy Tomskiy gosudarstvennyy universitet" na 2013-2020 gg. (2-y etap - 2015-2016 gg.) [The Roadmap of the Federal State Autonomous Institution of Higher Education "National Research Tomsk State University" for 2013-2020. (2nd stage: 2015-2016)]. Tomsk: Tomsk State University. [Online] Available from: viu.tsu.ru.

4. Demin, V.V. \& Sukhanova, E.A. (2015) Setevoe vzaimodeystvie klassicheskogo issledovatel'skogo universiteta i sistemy obshchego obrazovaniya: opyt i perspektivy [Networking of a classic research university and general education: experience and prospects]. Informatika $i$ obrazovanie. 6. pp. 3-6.

5. Prozumentova, G.N. (2012) Interaction potential of universities and schools: empirical models. Vestnik Tomskogo gosudarstvennogo universiteta Tomsk State University Journal. 358. pp. 182-187. (In Russian). 